\title{
The MdWRKY31 transcription factor binds to the MdRAV1 promoter to mediate ABA sensitivity
}

Xian-Yan Zhao', Chen-Hui Qi ${ }^{2}$, Han Jiang ${ }^{1}$, Chun-Xiang Yoư ${ }^{2}$, Qing-Mei Guan ${ }^{1}$, Feng-Wang Ma', Yuan-Yuan Li ${ }^{2}$ and Yu-Jin $\mathrm{HaO}^{2}$

\begin{abstract}
The phytohormone abscisic acid (ABA) is a major element involved in apple (Malus domestica) production because of its role in seed germination and early seedling development. The WRKY family, which is one of the largest families of transcription factors, plays an important role in ABA signaling in plants. However, the underlying molecular mechanisms of WRKY-mediated ABA sensitivity in apple are poorly understood. A genome-wide transcriptome analysis indicated that MdWRKY31 is a key factor induced by ABA. Quantitative real-time PCR showed that MdWRKY31 is induced by ABA in response to PEG4000, which is used to simulate drought. As a transcription factor, MdWRKY31 is localized in the nucleus. Ectopic expression of MdWRKY31 in Arabidopsis and Nicotiana benthamiana enhanced plant sensitivity to ABA. Overexpression of MdWRKY31 in apple roots and apple calli increased sensitivity to ABA, whereas repression of MdWRKY31 reduced sensitivity to ABA in the roots of 'Royal Gala'. Electrophoretic mobility shift assays, chromatin immunoprecipitation PCR, and yeast one-hybrid assays indicated that MdWRKY31 directly binds to the promoter of MdRAV1. Expression analyses of transgenic apple calli containing MdWRKY31 and pMdRAV1::GUS implied that MdWRKY31 represses the expression of MdRAV1. We also found that MdRAV1 binds directly to the promoters of $M d A B / 3$ and $M d A B / 4$ and repressed their expression. Our findings reveal a new important regulatory mechanism of MdWRKY31-MdRAV1-MdABls in the ABA signaling pathway in apple.
\end{abstract}

\section{Introduction}

Apple fruit quality and yield are affected by cultivars and various environmental factors. Among the environmental factors, phytohormones greatly influence plant development and productivity and play vital roles in the chlorophyll content, alterations to nutrients and moisture, the accumulation of harmful substances, and insect pests. As central integrators, plant hormones are involved in complex developmental and stress-adaptive signaling

Correspondence: Yuan-Yuan Li (liyy0912@163.com) or

Yu-Jin Hao (haoyujin@sdau.edu.cn)

${ }^{1}$ State Key Laboratory of Crop Stress Biology for Arid Areas/Shaanxi Key Laboratory of Apple, College of Horticulture, Northwest A\&F University, Yangling 712100 Shaanxi, China

${ }^{2}$ National Key Laboratory of Crop Biology, College of Horticulture Science and Engineering, Shandong Agricultural University, Tai-An, Shandong 271018, China cascades throughout plant responses involving signaling of the phytohormone abscisic acid (ABA). ABA plays a vital role in the activation of plant cellular responses to stress and is a central regulator of growth inhibition ${ }^{1-3}$. ABA also influences plant growth and development. At the ripening stage of 'Orin' apples, $\mathrm{ABA}$ promotes the biosynthesis of aromatic esters related to ethylene ${ }^{4}$. Studies have also shown that ABA plays an important role in sugar accumulation in fleshy fruits ${ }^{5}$.

ABA is involved in plant growth via a highly complex network; ABA is mainly a signaling pathway component. In the past few decades, the mechanism of the ABA signaling has been clearly revealed. ABA receptors, which perceive the initial activity, sense the ABA signal and elicit downstream signaling cascades to evoke physiological responses. ABA receptors include plasma membrane and 
intracellular receptors ${ }^{6-8}$. An unconventional G proteincoupled receptor (GCR2) and a novel class of G proteincoupled receptors (GTG1 and GTG2), which are plasma membrane ABA receptors, have been identified ${ }^{9-11}$. PYR/ PYL/RCAR proteins, which are considered cytosolic ABA receptors, mediate downstream gene expression of the ABA signaling pathway by directly inhibiting type $2 \mathrm{C}$ protein phosphatases ${ }^{12-14}$. Because of the complex network of ABA signaling, the functions of additional proteins in the ABA signaling pathway remain unclear.

As one of the largest transcription factor families, the WRKY family of proteins is associated with many stress response defense pathways because of the highly conserved WRKY domain of these proteins that binds the Wbox $(\mathrm{T})(\mathrm{T}) \mathrm{TGAC}(\mathrm{C} / \mathrm{T})^{15,16}$. WRKY transcription factors are involved in the ABA signaling pathway. AtWRKY40 is related to the $A B A$ signal by negatively regulating the expression of $A B I 4$ and $A B I 5$, which encode APETALA2 domain-containing and basic leucine zipper (bZIP)-type transcription factors, respectively ${ }^{17,18}$. During seed germination and early seedling development, abi4 and abi5 mutants exhibit ABA-insensitive phenotypes ${ }^{17}$. AtWRKY18 and AtWRKY60, which belong to the same group as AtWRKY40, play negative roles in ABA signaling $^{19}$. During seed germination and vegetative growth, AtWRKY63 and wrky2 mutants exhibit similar hypersensitive symptoms in response to exogenous $A B A^{20,21}$. In seed dormancy, AtWRKY41 participates in ABA signaling by regulating the direct expression of $A B I 3^{22}$. However, AtWRKY8 protects plants against TMV-cg by affecting ethylene and ABA signaling ${ }^{23}$.

Recently, AtWRKY6 was found to be a positive regulator of $\mathrm{ABA}$ signaling by directly regulating $R A V 1$ expression during seed germination and early seedling development ${ }^{24}$. In Arabidopsis, AtWRKY6 is induced significantly by $\mathrm{ABA}^{24}$, which clusters into the same group as does AtWRKY $31^{15}$. AtRAV1 overexpression slows rosette leaf and lateral root development, whereas suppressing AtRAV1 expression results in an earlyflowering phenotype ${ }^{25}$. AtRAV1 negatively regulates plant development and positively regulates leaf senescence by causing a premature phenotype ${ }^{26}$. AtRAV1 thus plays a vital role in $\mathrm{ABA}$ signaling by repressing the expression of $A B I 3, A B I 4$, and $A B I 5^{27}$.

Many WRKYs play crucial roles in response to $\mathrm{ABA}^{28}$; therefore, it is worthwhile to elucidate the functions and regulatory mechanisms of MdWRKYs in the ABA signaling pathway in apple. In this study, we found MdWRKY31 to be a positive regulator of ABA signaling. Ectopic expression of MdWRKY31 in Arabidopsis and Nicotiana benthamiana exhibited ABA-hypersensitive phenotypes during seed germination and early seedling development. When MdWRKY31 was overexpressed in apple seedlings and calli, the transgenic lines showed hypersensitive symptoms similar to those in response to ABA by elevating ABA-responsive genes. Correspondingly, repressing the expression of MdWRKY31 reduced sensitivity to ABA. By binding directly to its promoter, MdWRKY31 was able to repress the expression of $M d R A V 1$. In addition, MdRAV1 could bind to the promoters of $M d A B I 3$ and $M d A B I 4$ to inhibit their expression directly. Our findings reveal the function and a novel molecular mechanism of MdWRKY31 in mediating the ABA signaling pathway in apple.

\section{Results \\ Expression analysis of MdWRKY31}

ABA is an important phytohormone that plays a crucial role in plant growth and development. To identify potential genes associated with ABA, we performed an RNA-seq analysis to examine the differentially expressed genes in 'Royal Gala' seedlings treated with $100 \mu \mathrm{M}$ ABA or in seedlings under normal conditions. The data revealed that many genes were either upregulated or downregulated (Table S2). Among them, an apple WRKY transcription factor (MD05G1349800) was identified to be apparently induced by ABA treatment; this transcription factors was labeled MdWRKY31. To further verify the RNA-seq results, the expression of MdWRKY31 in 'Royal Gala' seedlings treated with $100 \mu \mathrm{M} \mathrm{ABA}$ for $0,1,3,6,12$, and $24 \mathrm{~h}$ was measured. The results indicated that the transcription of MdWRKY31 was induced by ABA, peaking after $3 \mathrm{~h}$ of treatment and then declining gradually (Fig. 1a). We also examined the transcript level of MdWRKY31 under abiotic stress, including polyethylene glycol (PEG), low temperature, and salt $(\mathrm{NaCl})$ stress, and found that PEG4000 treatment also induced the expression of MdWRKY31 (Fig. 1b). However, MdWRKY31 expression was strongly suppressed by low temperature and salt treatment (Fig. 1c, d). These results suggest that MdWRKY31 responded to various stresses and might function during these processes.

\section{Isolation and analysis of MdWRKY31}

To verify the function of MdWRKY31, RNA was extracted from tissue-cultured 'Royal Gala' seedlings. A cDNA template was obtained from RNA reverse transcription. The full-length sequence of $1821 \mathrm{bp}$ of MdWRKY31 was amplified with upstream and downstream primers of MdWRKY31 (MdWRKY31F/R). MdWRKY31 encodes a protein of 607 amino acid residues with a complete ORF and an isoelectric point of 7.19. Sequence analysis between MdWRKY31 and 71 WRKYs in Arabidopsis showed that MdWRKY31 clustered in a group together with AtWRKY42, AtWRKY47, AtWRKY31, and AtWRKY6 (Fig. 2a). Multiple sequence alignment of WRKY31 from Arabidopsis, Pyrus bretschneideri, and Malus domestica indicated that the MdWRKY31 protein contained a 

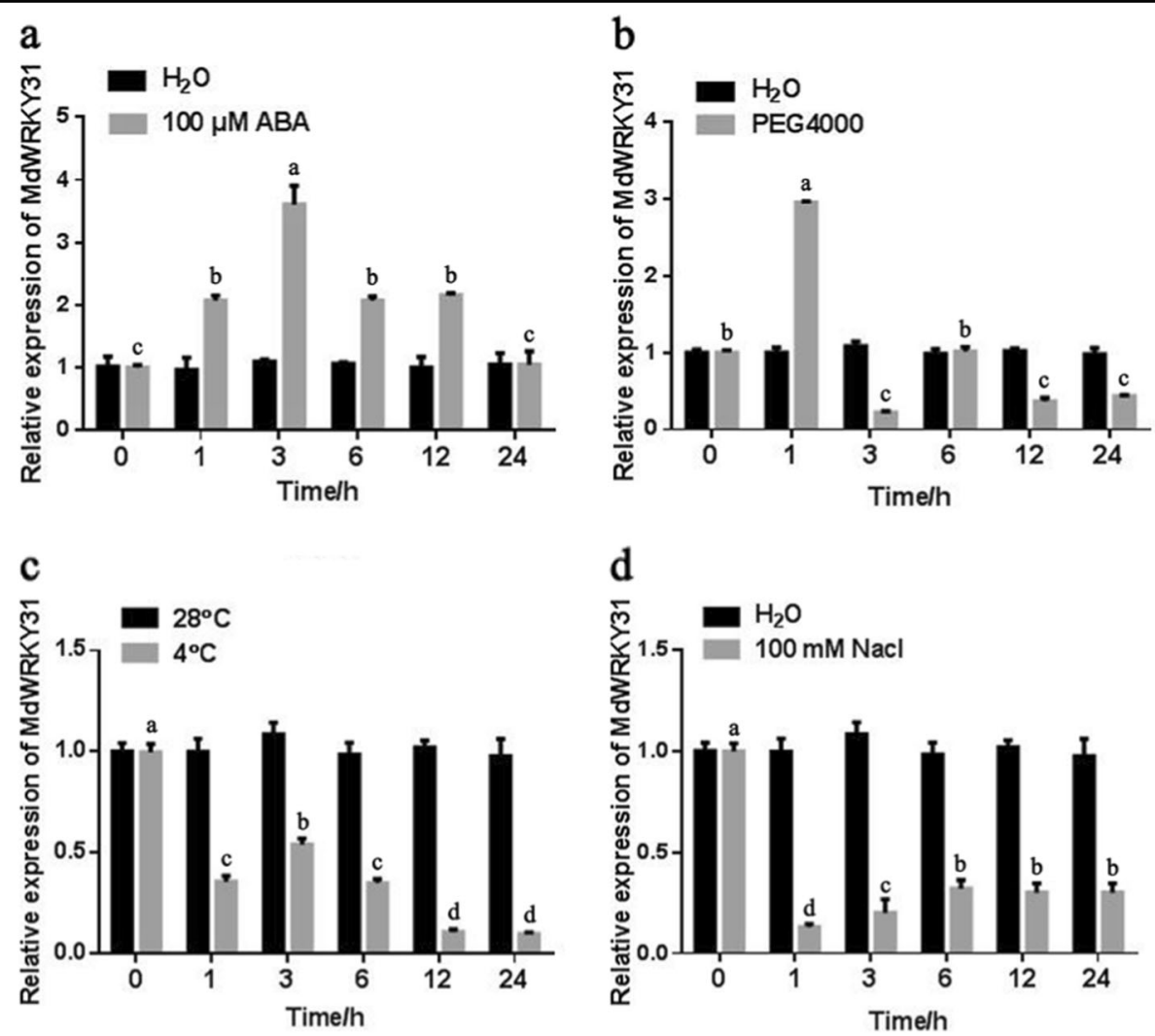

Fig. 1 Expression pattern of MdWRKY31 in response to abiotic stresses. a-d MdWRKY31 transcript levels in tissue cultures of 'Royal Gala' treated with $100 \mu \mathrm{M} \mathrm{ABA}, \mathrm{PEG} 4000,4^{\circ} \mathrm{C}$, and $100 \mathrm{mM} \mathrm{NaCl}$, respectively. $\mathrm{H}_{2} \mathrm{O}\left(\mathbf{a}, \mathbf{b}\right.$, d) treatment and $28^{\circ} \mathrm{C}(\mathbf{c})$ were used as controls; MdACTIN was an internal reference. The data were replicated three times, and the bars indicate SEs. Mean differences are significant at the $P_{0.05}$ level between the bars with different letters and not significant at the $P_{0.05}$ level with the same letters. The same setup applies to the following figure

conserved WRKY domain in its C-terminal region (Fig. 2b). An evolutionary tree was subsequently constructed to analyze MdWRKY31 and other WRKYs from different plant species, including Malus hupehensis, Pyrus bretschneideri, Prunus avium, Prunus mume, Prunus persica, Fragaria vesca subsp. Vesca, Ziziphus jujube, Morus notabilis, and Juglans regia. Two apple WRKY TFs from Malus domestica and Malus hupehensis demonstrated more than 99\% sequence similarity. The apple MdWRKY31 protein exhibited the closest relationship to the pear WRKY31 protein and the farthest relationship with the walnut WRKY31 protein (Fig. 2c).

\section{MdWRKY31 localizes to the nucleus}

Protein function is closely related to cellular location. To examine the cellular localization of the MdWRKY31 protein, a MdWRKY31-mCherry fusion protein whose $\mathrm{C}$-terminal expressed an enhanced red fluorescent protein (ERFP) was constructed. A plasmid containing RFP alone was used as a control. After transient injection into Nicotiana benthamiana, red fluorescence of MdWRKY31mCherry was observed in the nucleus, whereas the fluorescence of the control RFP was uniformly distributed across a greater field of vision; thus, MdWRKY31 was localized to the nucleus (Fig. 3).

\section{Ectopic expression of MdWRKY31 in Arabidopsis and tobacco increases sensitivity to ABA}

To determine the function of MdWRKY31, transgenic Arabidopsis and Nicotiana benthamiana plants overexpressing MdWRKY31 were obtained (Fig. 4a; Fig. S1). Seven transgenic Arabidopsis plants and five transgenic tobacco plants overexpressing MdWRKY31 presented different MdWRKY31 expression levels. Three transgenic lines (OE-1, OE-2, and OE-3 in Arabidopsis; 35:: MdWRKY31-1, 35::MdWRKY31-2 and 35::MdWRKY313 , respectively) with different $M d W R K Y 31$ gene expression levels (low, moderate, and high, respectively) were selected to perform subsequent phenotypic experiments involving ABA responses. Both the Arabidopsis and tobacco transgenic lines had a lower seed germination rate than did the wild type in response to $\mathrm{ABA}$ (Figs. 4b-e and 5a, b). With higher ABA concentrations, seeds of Arabidopsis and tobacco germinated relatively later. Root growth in response to ABA was subsequently examined in WT and MdWRKY31-overexpressing 


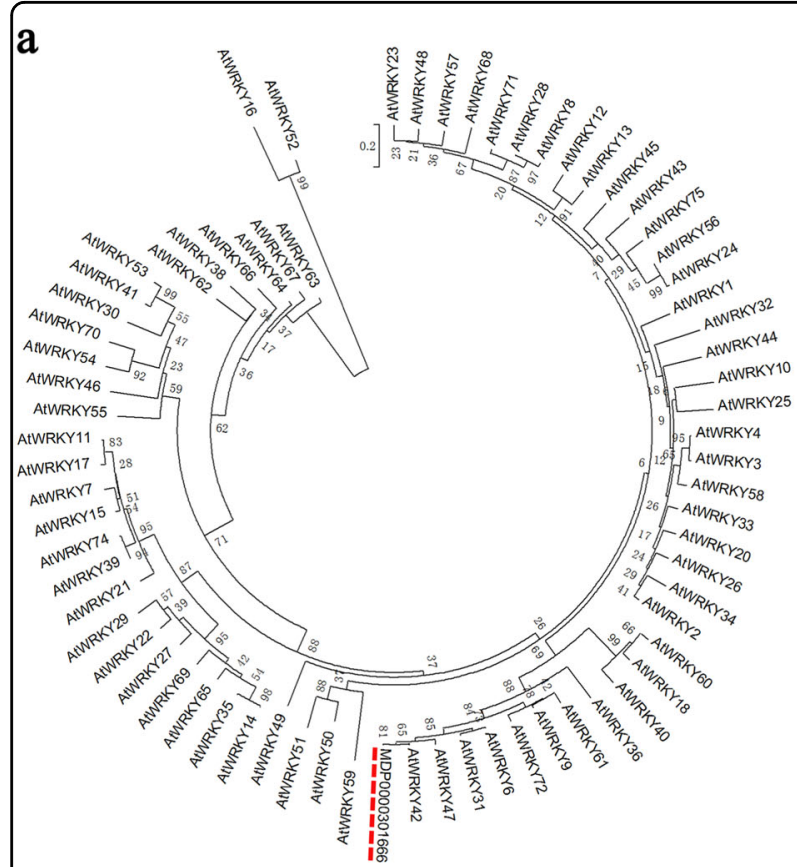

\section{b}
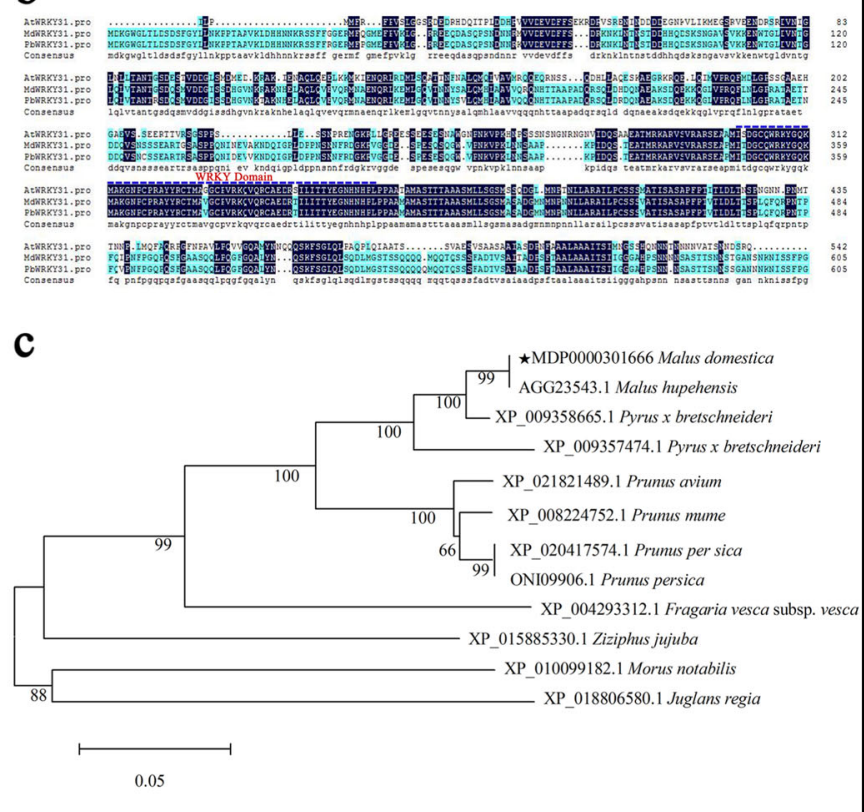

Fig. 2 Sequence analysis of the MdWRKY31 protein. a Multiple sequence alignment of MdWRKY31 and WRKYs from Arabidopsis. b Alignment of three WRKY proteins. WRKY domains are indicated by dotted lines. At: Arabidopsis thaliana, Md Malus domestica, Pb Pyrus bretschneideri. c Multiple sequence alignment of MdWRKY31 and WRKYs from other plant species
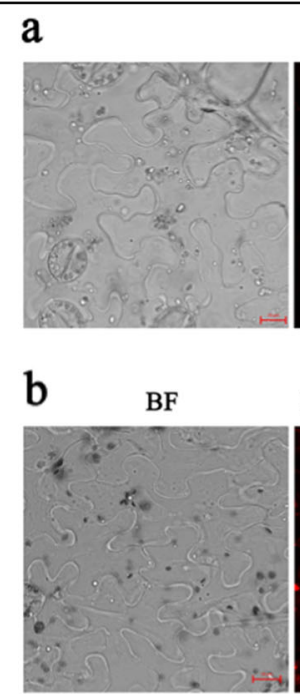

BF

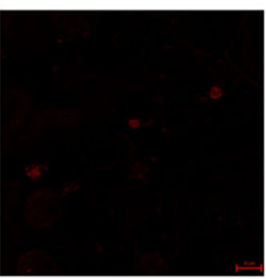

MdWRKY31-mCherry

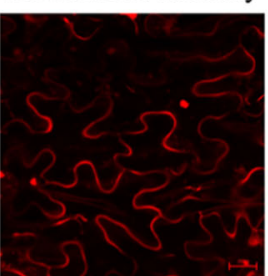

mCherry
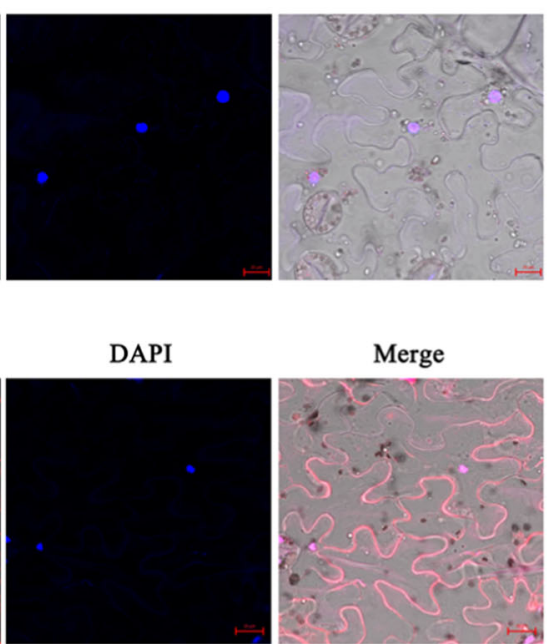

DAPI

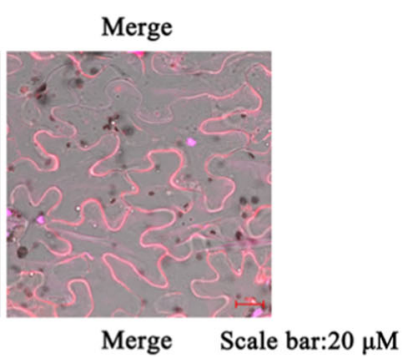

Fig. 3 Subcellular localization of the MdWRKY31 protein. Laser-scanning confocal microscopy images of Nicotiana benthamiana leaves expressing MdWRKY31-RFP. The micrographs show MdWRKY31-RFP localized to the nucleus. An empty RFP was used as a control. DAPI is a nuclear dye. The scale bar represents $20 \mu \mathrm{M}$

Arabidopsis and tobacco. We found the primary root length to be much shorter in the transgenic lines compared to the wild-type lines in both Arabidopsis and tobacco (Figs. 4f, g and 5c, d). These findings suggested that MdWRKY31 increased sensitivity to ABA in Arabidopsis and Nicotiana benthamiana.
MdWRKY31 positively regulates $A B A$ sensitivity in apple

$\mathrm{ABA}$ perception and signaling are major factors limiting apple production. To test whether MdWRKY31 was involved in the response to $\mathrm{ABA}$ in apple, $M d W R K Y 31$ was first fused to the expression vector PRI-GFP along with the strong $35 S$ promoter upstream. The 35S::MdWRKY31-GFP 


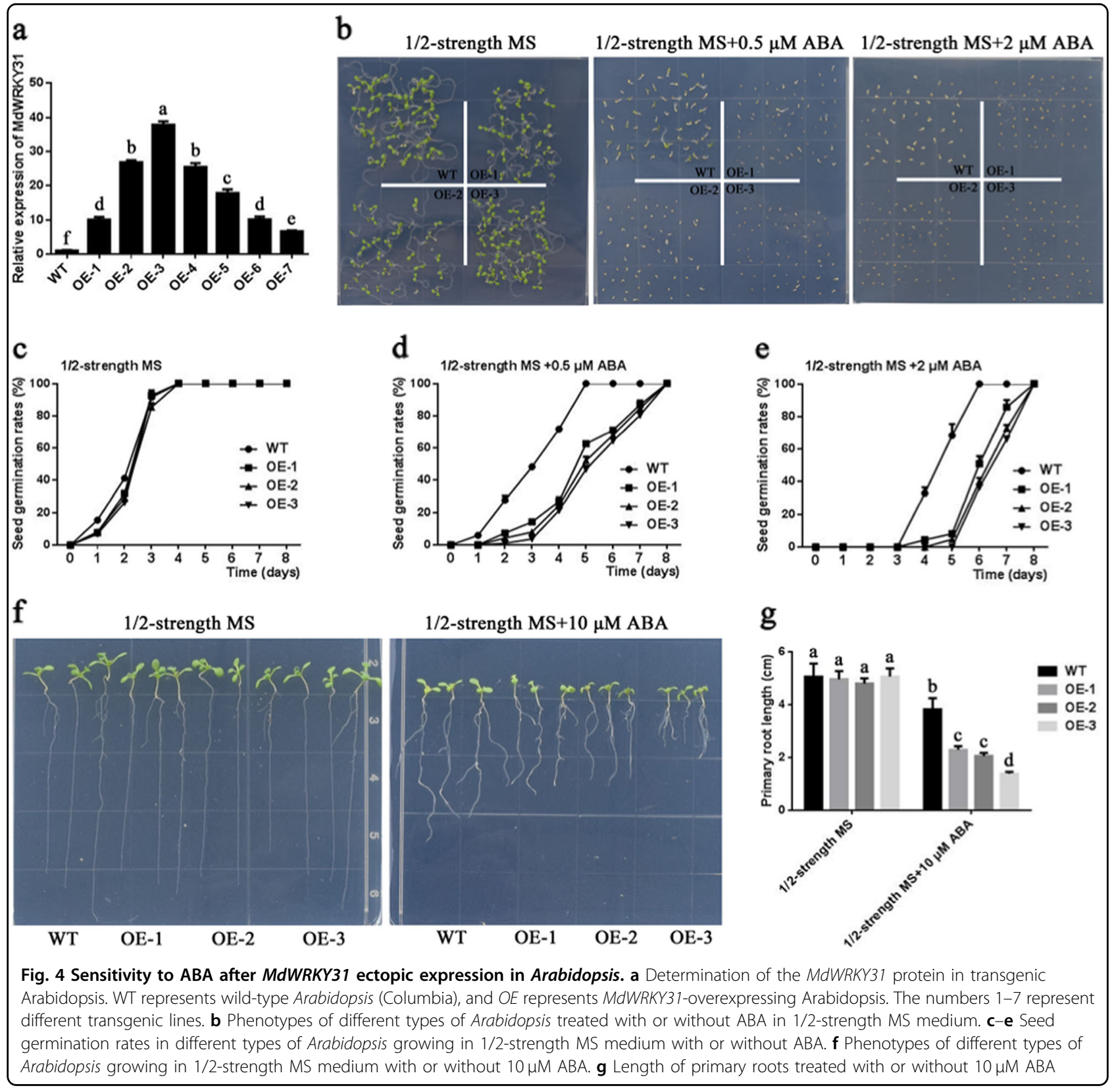

construct was then transformed into the roots of 'Royal Gala' seedlings using the Agrobacterium rhizogenes-mediated transformation method. Second, the specific fragment of MdWRKY31 fused into the expression vector pK7GWIWG2 along with an ERFP in its C-terminal region, after which the vector was transformed into the roots of 'Royal Gala' seedlings via the same transformation method. We then examined the fluorescence intensity and expression pattern of MdWRKY31. The results showed that the expression of MdWRKY31 was upregulated in MdWRKY31overexpressing apple roots (Fig. 6a, b) but noticeably downregulated in MdWRKY31 RNAi-transformed roots (Fig. 6c, d). The wild type and three transgenic lines with different MdWRKY31 expression levels were subsequently treated with $100 \mu \mathrm{M}$ ABA for 10 days. We evaluated the phenotypes of the WT and MdWRKY31 overexpressing/ RNAi lines and found that the MdWRKY31 OE seedlings exhibited more sensitivity to ABA than did the wild type; by contrast, downregulating MdWRKY31 expression reduced plant ABA sensitivity (Figs. 7a and 8a). Tetranitroblue tetrazolium chloride (NBT) staining indicated that MdWRKY31 overexpression produced higher superoxide ion $\left(\mathrm{O}^{2-}\right)$ than did the WT, and MdWRKY31 RNAi lines accumulated less $\mathrm{O}^{2-}$ than did the WT (Figs. 7b and 8b). In addition, three $M d W R K Y 31$-overexpressing lines contained more MDA, lower RWC, and less chlorophyll than did the 


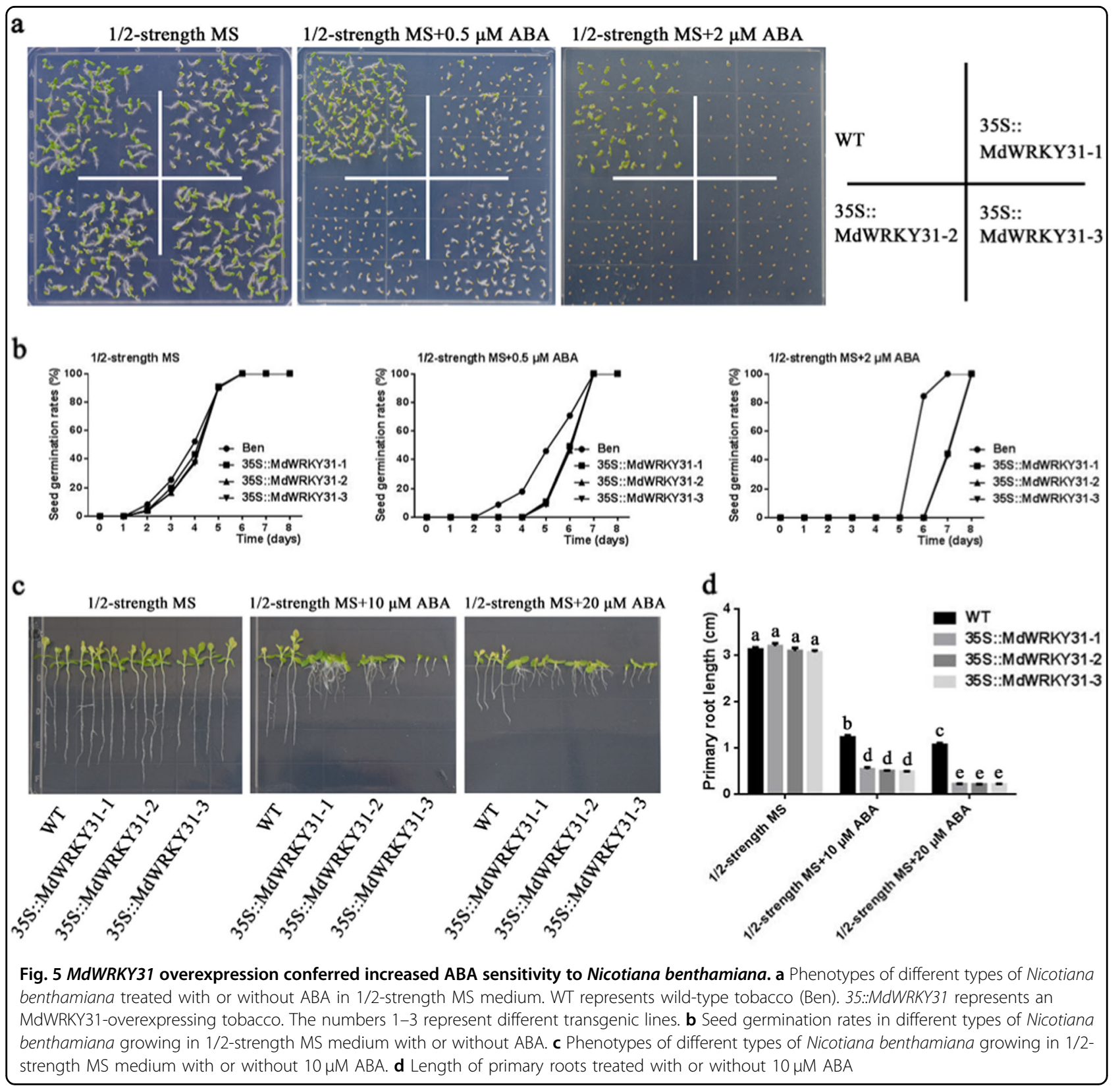

WT controls (Fig. 7c-e). These related physiological indicators of the ABA response were also detected in the leaves of MdWRKY31 RNAi lines; the MdWRKY31 RNAi seedlings accumulated less MDA and more chlorophyll and had a higher RWC than did the WT (Fig. 8c-e). MdWRKY31 transgenic apple roots exhibited great differences in ABA resistance; therefore, four ABA-responsive genes (MdAD29A, MdRAB18, MdEM1, and MdEM6) were measured. The results suggest that the expression of three of those genes (MdRAB18, MdEM1, and MdEM6) had varying degrees of increase in the $M d W R K Y 31$-overexpressing lines compared to the WT controls (Fig. 7f). Interestingly, compared to the WT controls, MdWRKY31-repressing lines
(RNAi-6 and RNAi-9) also exhibited slightly higher expression of the abovementioned genes (MdRAB18, $M d E M 1$, and MdEM6) (Fig. 8f). These results indicate that MdWRKY31 regulates ABA sensitivity in apple roots.

To confirm the function of MdWRKY31 in apple, we transformed 35S::MdWRKY31 to apple calli and generated six $M d W R K Y 31$ transgenic lines that present different MdWRKY31 expression levels (Fig. S2a). $M d W R K Y 31$ protein levels in the six transgenic lines were examined using an anti-GFP antibody. We found detectable MdWRKY31 proteins in all six transgenic calli (Fig. S2b). Next, three independent lines (OE-1, $\mathrm{OE}-2$, and $\mathrm{OE}-3$ ) were selected for subsequent 


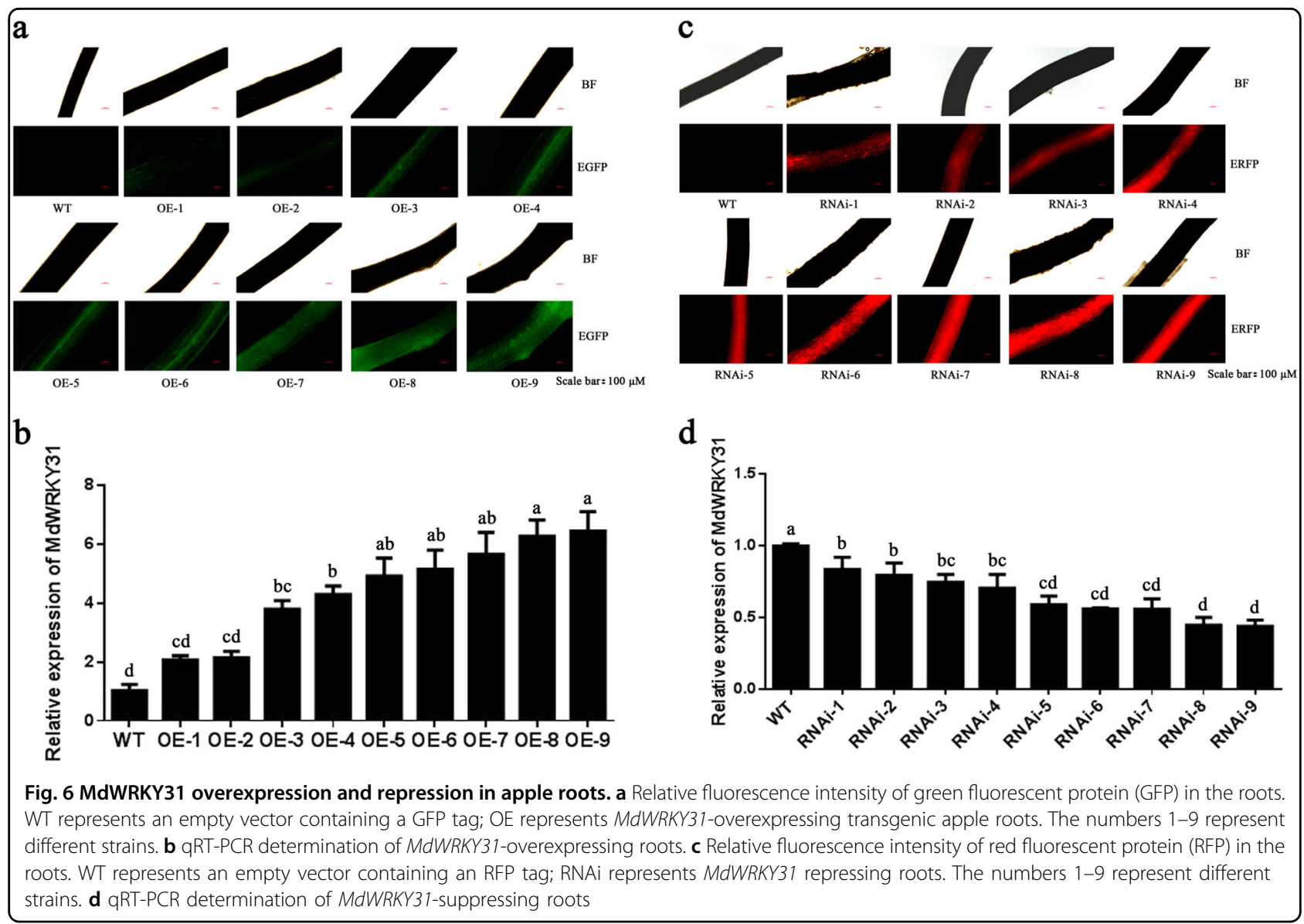

experiments. Apple calli were treated with 50, 100, and $150 \mu \mathrm{M} \mathrm{ABA}$. Evaluation of the phenotypes and fresh weight revealed that $\mathrm{ABA}$ at different concentrations inhibited apple callus growth and that the degree of inhibition was more obvious in the transgenic calli than in the WT calli (Fig. S2c, d). These results further confirm that MdWRKY31 acts as a positive regulator in the ABA signaling pathway in apple.

\section{MdWRKY31 represses the transcription of MdRAV1 by binding directly to its promoter in apple}

To explore the mechanism of MdWRKY31-regulated ABA resistance, related genes associated with ABA signaling were monitored in WT and MdWRKY31-overexpressing apple roots (Fig. S3). Many genes were upregulated and downregulated to varying degrees in $M d W R K Y 31$ OE roots compared to WT roots. Interestingly, the expression of two $M d R A V$ genes, MdRAV1 (MD16G1047700) and MdRAV2 (MD13G1046100), was clearly suppressed in the MdWRKY31 OE roots. The promoters of the $M d R A V 1$ and $M d R A V 2$ genes were analyzed, and one W-box motif, a WRKY binding motif, was identified in both promoter sequences (Table S3).
Therefore, we deduced that MdWRKY31 binds to the promoters of $M d R A V \mathrm{~s}$. First, an electrophoretic mobility shift assays (EMSA) was conducted to detect the binding of MdWRKY31 to the promoter regions of MdRAV1 or $M d R A V 2$. The results indicated that MdWRKY31 binds directly to the promoter of $M d R A V 1$ but not to the promoter of MdRAV2 (Fig. S4a) in vitro (Fig. 9a). When the TTGACC cis-element was replaced with the TACGTC element, the binding stopped (Fig. 9a), indicating specific binding. Yeast one-hybrid and chromatin immunoprecipitation (ChIP) experiments then confirmed the interaction between MdWRKY31 and the MdRAV1 promoter (Fig. 9b-d).

These results demonstrate that MdWRKY31 binds to the promoter of MdRAVI. To further examine whether the transcriptional activity of $M d R A V 1$ was induced or suppressed by MdWRKY31, a GUS reporter gene was fused downstream from the MdRAV1 promoter and labeled $p M d R A V 1:: G U S$. Additionally, we transformed pMdRAV1::GUS into apple calli and obtained $p M d R A V 1::$ GUS-overexpressing apple calli. MdWRKY31 was then transiently transformed into $p M d R A V 1$ :GUS and $p 1300-$ GN::GUS transgenic calli. We compared the GUS activity 


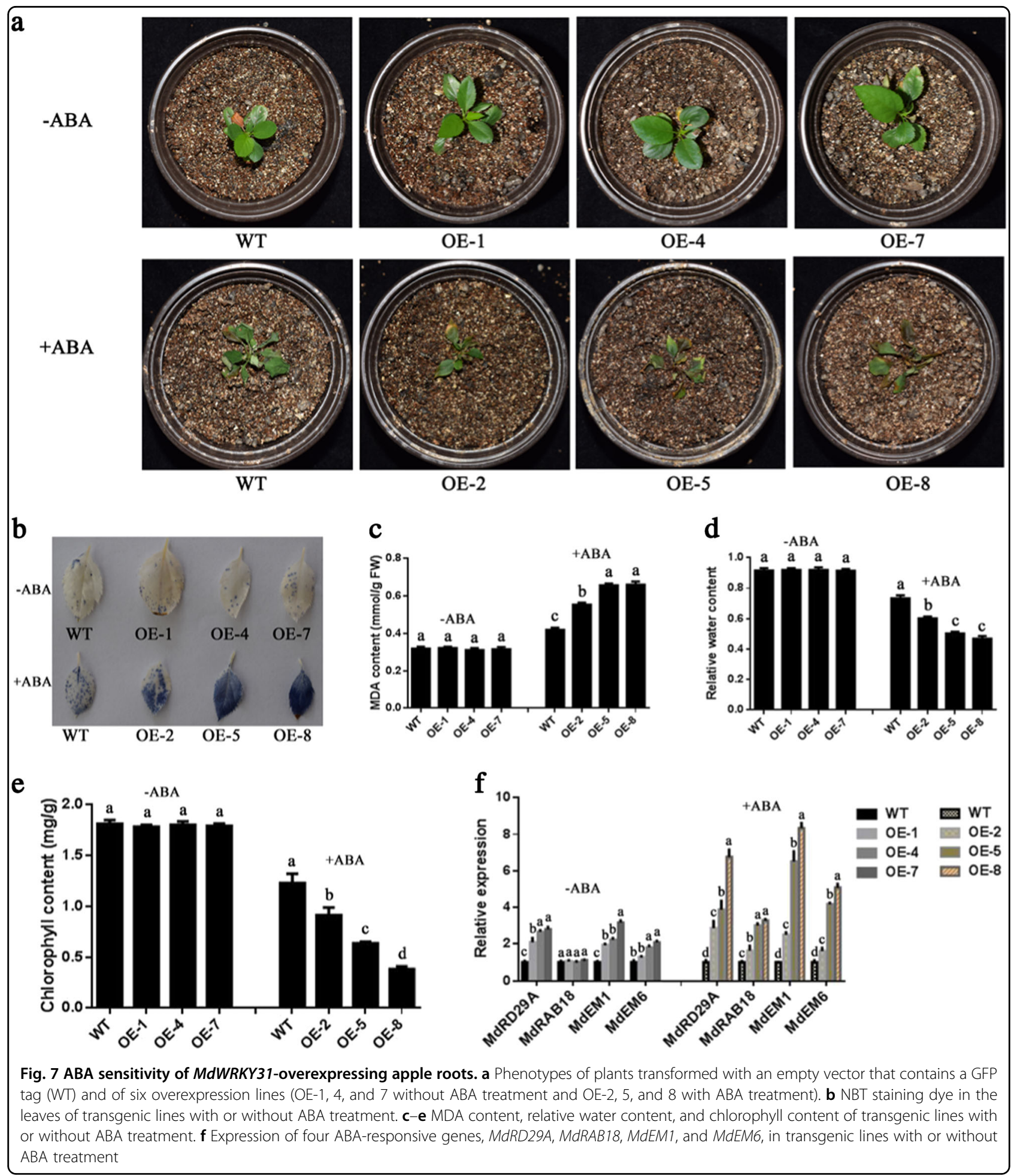

of the two transgenic calli and found that, compared with the GUS-overexpressing calli, the transgenic calli containing MdWRKY31 and $p M d R A V 1-G U S$ repressed the transcriptional activity of GUS (Fig. 9e). In general, the repression of MdRAV1 transcription in the MdWRKY31 transgenic calli and GUS staining results demonstrated that MdWRKY31 noticeably suppressed MdRAV1 expression (Fig. S3; Fig. 9e). Based on these findings, MdWRKY31 binds directly to the promoter regions of $M d R A V 1$ to repress its expression. 


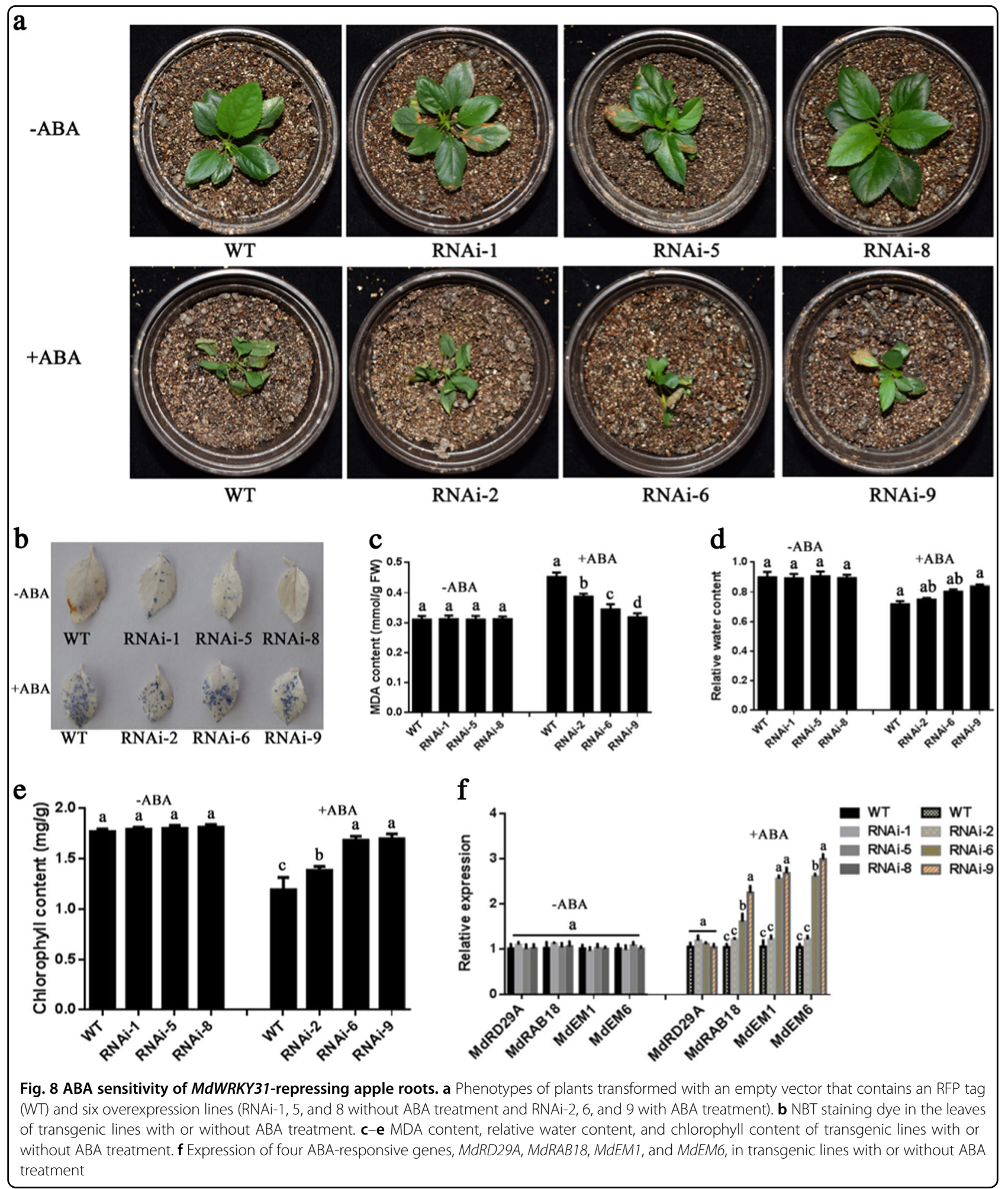

MdRAV1 directly binds to promoters of MdAB/s to repress their expression

As a transcription factor, AtRAV1 can bind to the promoters of $A t A B I 3, A t A B I 4$, and $A t A B I 5^{27}$. On the basis of the expression levels of MdABIs, the expression of $M d A B I 3, M d A B I 4$, and $M d A B I 5$ obviously increased in the MdWRKY31 OE lines compared to the WT (Fig. S3). We analyzed the promoter regions of the three $M d A B I$ 


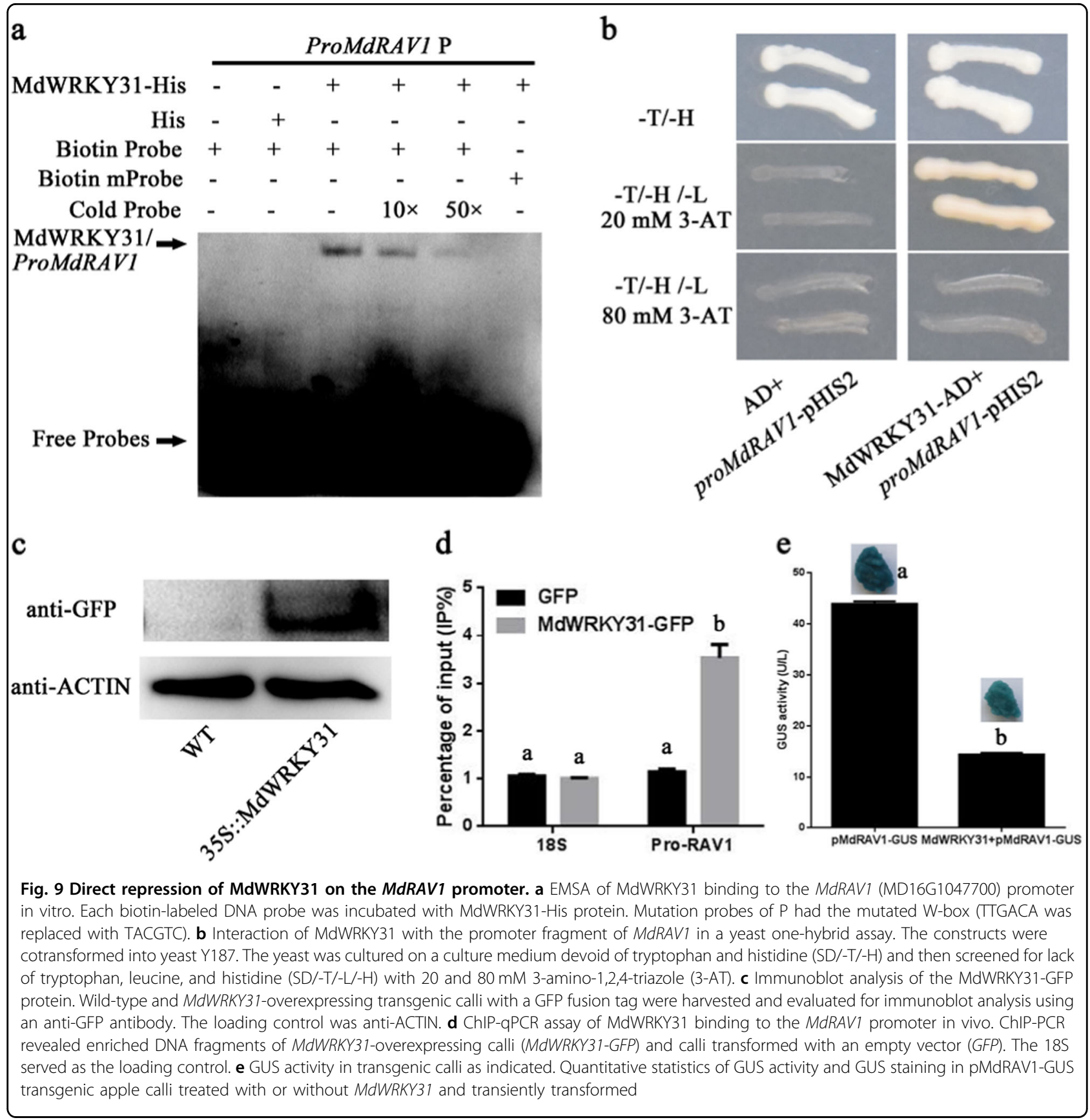

genes and found several conserved cis-acting elements (CAACA) in promoters of all three $M d A B I$ genes (Fig. S5). An EMSA assay was then carried out to detect interactions between MdRAV1 and the promoters of the $M d A B I$ genes. The results indicate that MdRAV1 could bind to CAACA at the P1 location of the promoters of $M d A B I 3$ and MdABI4 (Fig. 10a, b) but not MdABI5 in vitro (Fig. S4b). CAACA was subsequently mutated to TGGGG; binding was not detected (Fig. 10a, b), indicating specific binding. Yeast one-hybrid and ChIP assays then demonstrated the interaction between MdRAV1 and the $M d A B I 3$ and $M d A B I 4$ promoters (Fig. 10c).

To verify the suppression or activation of MdRAV1 on $M d A B I 3$ and MdABI4, the MdABI3 and MdABI4 promoters were fused to the GUS reporter gene downstream. We transformed $p M d A B I 3:: G U S$ and $p M d A B I 4:: G U S$ into apple calli and obtained $p M d A B I 3:: G U S$ and $p M d A B I 4::$ GUS transgenic calli. MdRAV1 was then transiently transformed into the $p M d A B I 3:: G U S$ and $p M d A B I 4:: G U S$ transgenic calli. GUS activities in different types of apple 


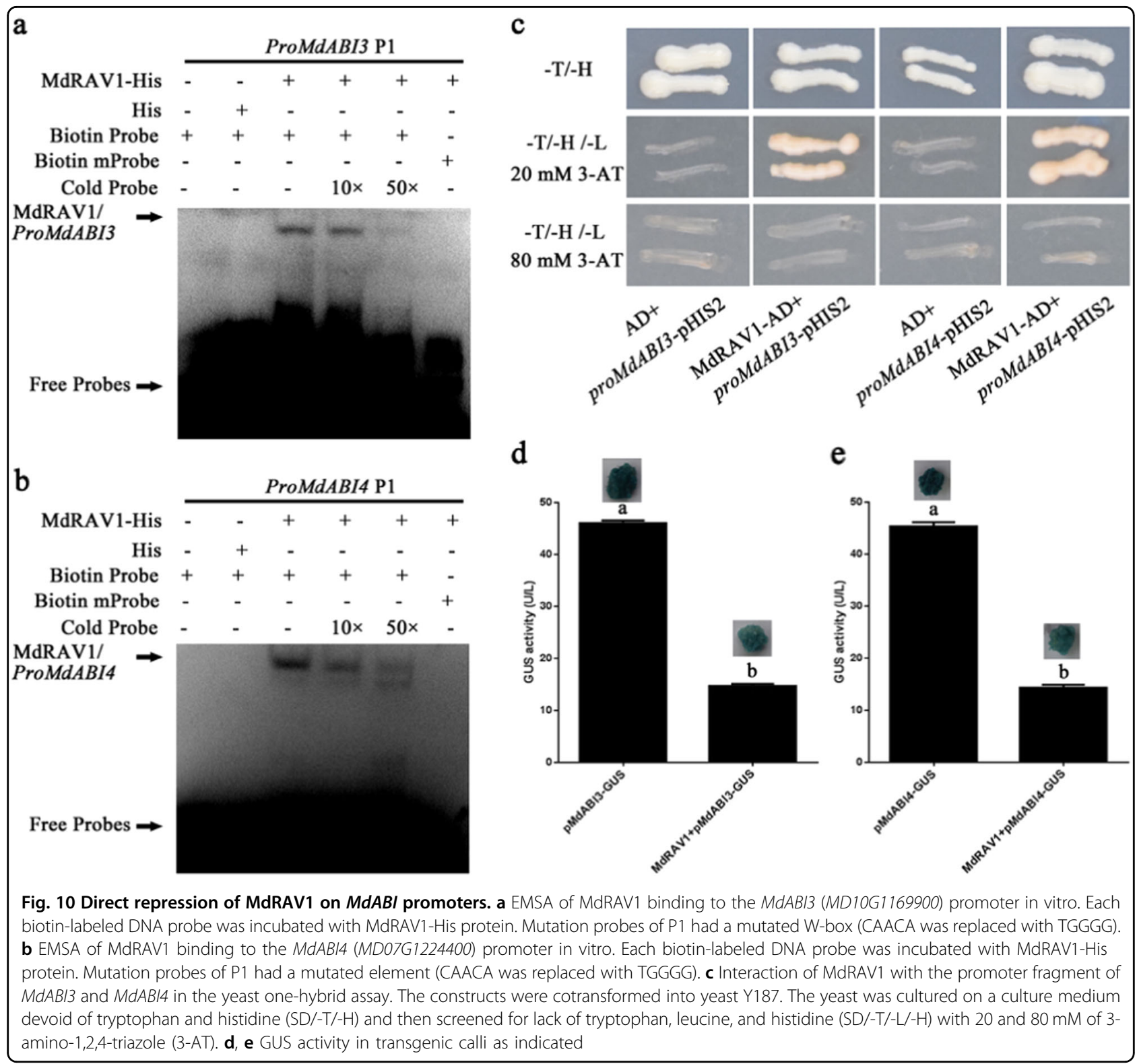

calli were detected, and the results show that MdRAV1 repressed the transcription activity of $p M d A B I 3:: G U S$ and pMdABI4::GUS (Fig. 10d). Therefore, MdRAV1 bound directly to the promoter regions of $M d A B I 3$ and $M d A B I 4$ to repress their expression.

\section{Discussion}

MdWRKY31 can be induced by ABA

$\mathrm{ABA}$ is one of the most common factors affecting apple quality and yield. In ABA signaling, the rapid accumulation of $\mathrm{ABA}$ in response to drought protects plants against damage $^{29}$. Many studies have shown that WRKYs play vital roles in ABA signaling and drought ${ }^{4,24,30}$. Transcriptome analysis has been indicated to be an effective method in biological research in recent years ${ }^{31-33}$. We used RNA-seq analysis to identify possible MdWRKYs that function in the ABA signaling pathway. Fortunately, one apple WRKY TF, MdWRKY31, was screened and found to be induced by ABA (Table S2). Further quantitative analysis revealed that ABA and PEG4000 (for simulating drought) significantly induced the expression of MdWRKY31, suggesting that MdWRKY31 may be related to ABA signaling and drought. We also found that treatment with $\mathrm{NaCl}$ and $4{ }^{\circ} \mathrm{C}$ repressed $M d W R K Y 31$ expression, implying that MdWRKY31 is involved in various abiotic stresses.

\section{WRKY31 is conserved in different plant species}

WRKY proteins are often classified into groups according to the number of WRKY domains and the 
features of their zinc-finger-like motif ${ }^{15}$. MdWRKY31 belongs to Group II and is homologous to AtWRKY42, AtWRKY47, AtWRKY31, and AtWRKY6 in Arabidopsis. In soybean, GmWRKY31 is involved in SA-mediated immune responses by binding the cis-acting W-box element of the GmSAGT1 gene ${ }^{34}$. In rice, WRKY55/ WRKY31 positively regulates resistance to Magnaporthe oryza $^{35}$. The WRKY domain and phylogenetic tree analysis of WRKY31s implied that the function of WRKY31 could be conserved among different plant species.

\section{MdWRKY31 localizes to the cell nucleus}

Most transcription factors are located in the nucleus to perform their functions. OsWRKY67 positively regulates resistance against blast and bacterial blight and is ubiquitously expressed and sublocalized in the nucleus ${ }^{36}$. In grapevine, VaWRKY14 (involved in drought resistance) is localized to the nucleus ${ }^{37}$. A cucumber WRKY transcription factor, CsWRKY46, found exclusively in the nucleus, increases resistance to $\operatorname{cold}^{38}$. The location of MdWRKY31 indicates that it also is present in the cell nucleus, suggesting that MdWRKY31 potentially performs its function there.

\section{MdWRKY31 positively regulates $A B A$ signaling}

Many reports have found that WRKYs are involved in ABA signaling. In Arabidopsis, WRKY41 controls seed dormancy by directly regulating $A B I 3$ expression; WRKY40 directly represses the expression of the $A B I 5$ ABA-responsive gene ${ }^{19,22}$. WRKY6 positively regulates ABA signaling by directly inhibiting the expression of $R A V 1^{24}$. FvWRKY42 increases ABA sensitivity and improves osmotic stress resistance in strawberry ${ }^{30}$. In cotton, GhWRKY6-like improves salt tolerance by activating the $A B A$ signaling pathway ${ }^{39}$. We found that ectopic expression of MdWRKY31 in Arabidopsis and tobacco and its homologous expression in apple calli and roots can increase sensitivity to ABA; thus, MdWRKY31 may positively mediate ABA signaling in plants.

It is difficult to obtain transgenic apple lines with stable expression because of the immaturity of the apple transformation system and its characteristics as a perennial woody plant. However, Agrobacterium rhizogenes-mediated transformation into apple roots renders gene function identification possible ${ }^{40}$. In this study, the function of MdWRKY31 was confirmed in apple roots. Phenotypes of apple roots overexpressing and repressing MdWRKY31 indicated that MdWRKY31 functions as a positive regulator in the ABA signaling pathway. The ABAresponsive genes $R A B 18$ and $R D 29 B$ in Arabidopsis have always been used as indicators of the ABA signaling pathway ${ }^{41,42}$. EM1, EM6, and $R A B 18$ are three representative ABA-responsive genes. Analysis of the changes in the expression of these genes in transgenic
MdWRKY31 overexpression or suppression apple roots indicated that relevant research from Arabidopsis is applicable to other species, such as apple. The phenotypes of different MdWRKY31 transgenic materials (Arabidopsis, tobacco, apple calli, and apple roots) suggested that the function of MdWRKY31 in the ABA signaling pathway is conserved beyond plant species.

$\mathrm{O}^{2-}$, a form of ROS (reactive oxygen), is a toxic molecule that can cause oxidative damage to proteins, DNA, and lipids ${ }^{43}$. ABA induces the production of $\operatorname{ROS}^{44}$. In this study, MdWRKY31 overexpression in transgenic apple plants promoted the accumulation of $\mathrm{O}^{2-}$ in response to $\mathrm{ABA}$. The release of reactive oxygen species can cause chlorophyll degradation, programmed cell death, and MDA accumulation ${ }^{45,46}$. MdWRKY31 overexpression in transgenic plants subsequently produced a higher MDA content and less chlorophyll and RWC in those plants than in the WT controls in response to ABA treatment.

\section{MdWRKY31 can bind to the promoters of MdRAV1, which interact with the promoters of $M d A B I 3$ and MdABI4}

Many protein kinases and transcription factors are involved in $\mathrm{ABA}$ signaling. $\mathrm{ABI} 3, \mathrm{ABI} 4$, and $\mathrm{ABI} 5$ mediate seed dormancy and seedling development ${ }^{47-53}$, and ABF2, $\mathrm{ABF} 3$, and $\mathrm{ABF} 4$ promote chlorophyll degradation and leaf senescence through ABA signaling ${ }^{54}$. SnRK2 kinases (SRK2D/SnRK2.2, SRK2E/SnRK2.6 and SRK2I/SnRK2.3 in Arabidopsis) play vital positive roles in ABA signaling downstream of the pyrabactin resistance1/PYR1-like/ regulatory components of $\mathrm{ABA}$ receptor (PYR/PYL/ RCAR) proteins ${ }^{12,13,55,56}$. The Arabidopsis RAV1 transcription factor provides plant insensitivity to $\mathrm{ABA}^{27}$. $M d W R K Y 31$ overexpression alters the expression of these genes involved in ABA signaling. The repression of $M d R A V 1$ and increased expression of $A B F \mathrm{~s}, A B I \mathrm{~s}$, and SnRKs indicated that MdWRKY31 may be a positive regulator in ABA signaling. Studies have shown that AtWRKY42, which clustered in a group together with MdWRKY31, often functions as a transcriptional repressor $^{57}$. Therefore, we proposed that MdWRKY31 also acted as a repressor of downstream genes; the MdRAV genes, whose expression was downregulated in the MdWRKY31-overexpressing plants, were selected for further investigation of their interaction. Direct binding of MdWRKY31 to the promoter regions of MdRAV1 and the binding of MdRAV1 to the promoters of MdABI3 and MdABI4 subsequently revealed that MdWRKY31 participated in $A B A$ signaling by interacting directly with ABA-related genes to repress their transcription.

\section{Conclusions}

In conclusion, we identified the WRKY TF MdWRKY31 in apple, which was significantly induced by $A B A$. 
Compared with control plants, MdWRKY31 transgenic plant materials, including Arabidopsis, tobacco, and apple, exhibited greater ABA sensitivity, indicating that MdWRKY31 is a positive regulator in the ABA signaling pathway. Furthermore, we found that MdWRKY31 repressed the transcription of the $M d R A V 1$ gene by binding directly to promoter region of $M d R A V 1$. The results further revealed that MdRAV1 bound to the promoters of $M d A B I 3$ and $M d A B I 4$ and inhibited their expression. Our findings identified the function of MdWRKY31 in plants and the regulatory mechanism of MdWRKY31 to ABA sensitivity, which is useful in comprehending the complex TF-regulated network, and provide a potential gene for apple cultivar improvement. Rootstock improvement is also practical for apple, which is a grafted crop species. Apple root transformation may be a possible way to alter rootstock characteristics.

\section{Materials and methods}

\section{Plant materials and experimental treatments}

The 'Orin' callus cultivar was cultured on MS medium containing $0.5 \mathrm{mg} / \mathrm{L} \mathrm{2,4-dichlorophenoxyacetic} \mathrm{acid} \mathrm{(2,4-}$ D) and $1.5 \mathrm{mg} / \mathrm{L}$ 6-benzylaminopurine $(6-\mathrm{BA})$ at $26^{\circ} \mathrm{C}$ in the dark. Tissue cultures of the 'Royal Gala' cultivar in vitro were grown on MS medium supplemented with $1.5 \mathrm{mg} / \mathrm{L} 6$-BA and $0.2 \mathrm{mg} / \mathrm{L}$ IAA at $26^{\circ} \mathrm{C}$ under a $16-\mathrm{h}$ light/8-h dark photoperiod. The roots of 'Royal Gala' tissue-cultured seedlings were treated with water (control), $100 \mu \mathrm{mol} / \mathrm{L} \mathrm{ABA}, 4{ }^{\circ} \mathrm{C}$ temperature (low temperature), $100 \mu \mathrm{mol} / \mathrm{L} \mathrm{NaCl}$, or PEG4000 for $0,1,3,6,12$, and $24 \mathrm{~h}$. The samples were then quickly frozen in liquid nitrogen and stored in a refrigerator at $-80^{\circ} \mathrm{C}$.

Nicotiana benthamiana (Ben), wild-type Arabidopsis (COL), and transgenic Nicotiana benthamiana and Arabidopsis were screened by MS media with $60 \mathrm{mg} / \mathrm{L}$ kanamycin. Polymerase chain reaction (PCR) detected positive transgenic plants. After continuous screening for three generations, T3 homozygous plants were obtained and used for phenotypic experiments. Arabidopsis seeds were sown on MS medium with or without ABA for the germination experiments. Images were taken after 8 days of growth, after which the germination rate was analyzed. Four days after being sown, seedlings without ABA treatment were transferred to 1/2-strength MS media or 1/2-strength MS media with $10 \mu \mathrm{M}$ ABA. These culture dishes were placed in a growth chamber at $25^{\circ} \mathrm{C}$ under a $16-\mathrm{h}$ photoperiod. The root length of the seedlings was evaluated after 7 days, and the average value of 40 plants was counted. Transgenic apple lines were transferred to a nutrient-rich potting medium that mixed with vermiculite $(1: 1)$ and treated with $100 \mu \mathrm{M}$ ABA or no ABA for 10 days. Plant leaves were then used to analyze ABA-related indices, including the NBT dyeing, MDA content, relative water content, chlorophyll content, and relevant ABA-responsive genes.

\section{Subcellular localization analysis}

To explore the subcellular localization of MdWRKY31, the open reading frame (ORF) of MdWRKY31 was amplified from 'Royal Gala' apple tissue culture seedlings using PCR in conjunction with MdWRKY31-F and MdWRKY31-R as primers (Table S1). Based on $M d W R K Y 31$ sequences, the Gateway system was used to insert the MdWRKY31 ORF into the pENTR ${ }^{\mathrm{TM}}$ Directional TOPO vector with MdWRKY31-TOPO upstream and downstream primers. The recombination reaction (LR) was used to insert the MdWRKY31 gene into the PAL1107 vector to construct a MdWRKY31-RFP vector for subcellular localization.

Bacterial solutions of MdWRKY31-RFP and P19 were cultured for $12 \mathrm{~h}$. After centrifugation, the Agrobacterium liquid was suspended in an MMA liquid medium and mixed in a 1:1 proportion of MdWRKY31-RFP:P19 for $4-5 \mathrm{~h}$. A $500 \mu \mathrm{l}$ suspension liquid was injected into the leaves of Nicotiana benthamiana. Images were taken with a two-photon laser confocal microscope (Carl Zeiss;German) at 3-4 days after the plants were transferred to a growth chamber with a temperature of $25^{\circ} \mathrm{C}$ and a $16-\mathrm{h}$ photoperiod.

\section{Construction of vectors and obtained transgenic lines}

To construct MdWRKY31 overexpression vectors, the ORF of MdWRKY31 was digested with SalI/KpnI and cloned into GFP plant transformation vectors downstream of the CaMV $35 S$ promoter. The MdWRKY31GFP vector was then transformed into Agrobacterium rhizogenes pRi2659 (Weidi Biotechnology, Shanghai, China). According to the Agrobacterium conversion method, the MdWRKY31-GFP vector was successfully genetically introduced into the roots of apple plants.

A specific sequence of MdWRKY31 via primers MdWRKY31-FR-F and MdWRKY31-FR-R was inserted into the $\mathrm{pENTR}^{\mathrm{TM}}$ Directional TOPO vector. The LR was used to insert the specific fragment of MdWRKY31 into the pK7GWIWG2 vector to construct a MdWRKY31-RFP vector. The resulting MdWRKY31-RFP vector was genetically introduced into apple plant roots according to Agrobacterium rhizogenes MSU44-mediated transformation as described by Ma et al. ${ }^{40}$. The primers used in this paper are detailed in Table S1.

\section{Analysis of gene expression}

TRIzol reagent (Invitrogen, Carlsbad, CA) was used to extract the total RNA from 'Royal Gala' tissues (calli and seedlings), Arabidopsis, and Nicotiana benthamiana. cDNA synthesis was executed with a PrimeScript ${ }^{\mathrm{TM}} \mathrm{RT}$ reagent kit (TaKaRa, Dalian, China). Quantitative realtime PCR (qRT-PCR) was used to test the MdWRKY31 expression level in response to $\mathrm{ABA}, \mathrm{NaCl}, 4^{\circ} \mathrm{C}$, and PEG4000. In different transgenic Arabidopsis, Nicotiana 
benthamiana, and apple tissues, MdActin (GenBank accession number CN938024) was used as the reference gene.

For qRT-PCR, mixed solutions were executed with iQ SYBR Green Supermix in an iCycler iQ5 system (Bio-Rad, Hercules, CA, USA) according to the manufacturer's instructions. Analyses of specific mRNA levels were performed using relative quantification via the cycle threshold (Ct) 2- $\Delta \Delta \mathrm{Ct}$ method. Each assay was carried out for three biological replicates. PCR profiles were determined on the basis of the following protocol: preincubation at $95^{\circ} \mathrm{C}$ for $5 \mathrm{~min}$; 30 cycles of $95^{\circ} \mathrm{C}(30 \mathrm{~s}), 58^{\circ} \mathrm{C}(30 \mathrm{~s})$, and $72{ }^{\circ} \mathrm{C}(30 \mathrm{~s})$; and a $72{ }^{\circ} \mathrm{C}(5 \mathrm{~min})$ final extension. The corresponding primers used are listed in Table S1.

\section{Determination of the relative water content and chlorophyll content}

The relative water content (RWC) was determined based on the method described by Ma et al. ${ }^{40}$ as follows: $\mathrm{RWC}=$ (fresh weight - dry weight) $/$ (rehydrated weight dry weight).

Similarly, the chlorophyll content was measured according to the method described by An et al. ${ }^{58}$ as follows:

$$
\begin{aligned}
& \mathrm{C}_{\mathrm{a}}=13.95 \mathrm{D}_{665}-6.88 \mathrm{D}_{649} \\
& \mathrm{C}_{\mathrm{b}}=24.96 \mathrm{D}_{649}-6.88 \mathrm{D}_{665}
\end{aligned}
$$$$
\text { Chlorophyll content }=\mathrm{C}_{\mathrm{a}}+\mathrm{C}_{\mathrm{b}}
$$

\section{MDA content and NBT staining}

As described by Ma et al. ${ }^{40}$, the absorbance at 450, 532, and $600 \mathrm{~nm}$ was measured with an UV/vis spectrophotometer (UV-2450). MDA levels were calculated as follows: MDA content (mmol/g FW) 1/4[6.542*(OD532 -OD600)-0.559*OD450] (mmol/L)*V (ml)/fresh weight (g FW).

Leaves soaked in NBT staining buffer $(0.5 \mathrm{mg} / \mathrm{ml})$ were vacuum treated for $20 \mathrm{~min}$ for better dyeing. The samples were then stained for $8 \mathrm{~h}$ in darkness at $28^{\circ} \mathrm{C}$ and then boiled for $5 \mathrm{~min}$ with ethanol:lactic acid:glycerin (3:1:1) in a fixative solution until the chlorophyll was removed and the samples cooled. Anhydrous alcohol was then added prior to observations.

\section{GUS analysis}

Apple calli were used for transient expression assays. Wild-type promoters of $M d R A V 1, M d A B I 3$, and MdABI4 were cloned into $1300-G N$, which was fused to the GUS reporter gene. The constructed $p M d R A V 1:: G U S$ plasmids (the $p M d A B I 3:: G U S$ plasmid and $p M d A B I 4:: G U S$ plasmid) were transformed into apple calli via the Agrobacterium-mediated method. 35S::MdWRKY31 (35S:: $M d R A V 1)$ was then cotransformed into the $p M d R A V 1::$ GUS (pMdABI3::GUS and pMdABI4::GUS) and p1300GN::GUS transgenic calli. Finally, histochemical staining was conducted to measure the GUS activity in the treated calli using the method described by Zhao et al. $(2016)^{59}$.

\section{Chromatin immunoprecipitation (ChIP) qPCR analysis}

The 35::MdWRKY31::GFP and 35::GFP transgenic calli were used for a ChIP assay. An anti-GFP antibody (Beyotime, Haimen, China) was applied to ChIP-qPCR as described by $\mathrm{Hu}$ et al. $^{60}$. Immunoprecipitated samples were used as templates for the qPCR assay with primers listed in Table S1.

\section{Electrophoretic mobility shift assays (EMSAs)}

An EMSA was performed as described by Xie et al. ${ }^{61}$. $M d W R K Y 31$ and MdRAV1 were cloned into the expression vector $p E T-32 a(+)$. The MdWRKY31-HIS and MdRAV1-HIS recombinant proteins were expressed in Escherichia coli strain BL21 and purified using a His Microspin Purification kit (Tiangen, Beijing, China). The oligonucleotide probes of the MdRAV1, MdABI3, and $M d A B I 4$ promoters were labeled by a company (Sangon Biotech, Beijing, China). The binding specificity was determined by measuring its competition with excess unlabeled oligonucleotides. The primers used are listed in Table S1.

\section{Statistical analysis}

Appropriate methods using R (3.0.2) with the R Commander package were employed to analyze three parallel experiments statistically. Mean differences between the bars are significant at the $P_{0.05}$ level for different letters but not significant at the $P_{0.05}$ level for the same letters.

\section{Acknowledgements \\ This work was supported by grants from the National Natural Science Foundation of China (U1706202, 31772275), the Natural Science Fund for Excellent Young Scholars of Shandong Province (ZR2018JL014) and the Ministry of Education of China (IRT15R42).}

\section{Author details}

${ }^{1}$ State Key Laboratory of Crop Stress Biology for Arid Areas/Shaanxi Key Laboratory of Apple, College of Horticulture, Northwest A\&F University, Yangling 712100 Shaanxi, China. ${ }^{2}$ National Key Laboratory of Crop Biology, College of Horticulture Science and Engineering, Shandong Agricultural University, Tai-An, Shandong 271018, China

\section{Authors contributions}

Y.-J.H. and Y.-Y.L. conceived the study; X.-Y.Z. and C.-H.Q. conducted the experiments; X.-Y.Z., Q.-M.G., F.-W.M., and H.J. analyzed the data; and X.-Y.Z., Y.-Y.L., and Y.-J.H. wrote the manuscript. All the authors read and approved the final manuscript.

Conflict of interest

The authors declare that they have no conflict of interest.

\section{Publisher's note}

Springer Nature remains neutral with regard to jurisdictional claims in published maps and institutional affiliations. 
Supplementary Information accompanies this paper at (https://doi.org/ 10.1038/s41438-019-0147-1).

Received: 20 December 2018 Revised: 29 March 2019 Accepted: 31 March 2019

Published online: 01 June 2019

\section{References}

1. Cutler, S. R., Rodriguez, P. L., Finkelstein, R. R. \& Abrams, S. R. Abscisic acid emergence of a core signaling network. Annu. Rev. Plant Biol. 61, 651-679 (2010).

2. Raghavendra, A. S., Gonugunta, V. K., Christmann, A. \& Grill, E. ABA perception and signalling. Trends Plant Sci. 15, 395-401 (2010).

3. Weiner, J. J., Peterson, F. C., Volkman, B. F. \& Cutler, S. R. Structural and functional insights into core ABA signaling. Curr. Opin. Plant Biol. 13, 495-502 (2010).

4. Wang, S. et al. Abscisic acid is involved in aromatic ester biosynthesis related with ethylene in green apples. J. Plant Physiol. 221, 85-93 (2018).

5. Pan, Q. H. et al. Abscisic acid activates acid invertases in developing grape berry. Physiol. Plant. 125, 157-170 (2005).

6. Assmann, S. M. Ins and outs of guard cell ABA receptors. Plant Cell $\mathbf{6}$, 1187-1190 (1994)

7. Finkelstein, R. R. \& Lynch, T. J. The Arabidopsis abscisic acid response gene ABI5 encodes a basic leucine zipper transcription factor. Plant Cell 12, 599-609 (2000).

8. Verslues, P. E. \& Zhu, J. K. New developments in abscisic acid perception and metabolism. Curr. Opin. Plant Biol. 10, 447-452 (2007).

9. Liu, X. et al. AG protein coupled receptor is a plasma membrane receptor for the plant hormone abscisic acid. Science 315, 1712-1716 (2007).

10. Johnston, C. A. et al. Comment on a G protein coupled receptor is a plasma membrane receptor for the plant hormone abscisic acid. Science $\mathbf{3 1 8}$ 914-914 (2007)

11. Pandey, S., Nelson, D. C. \& Assmann, S. M. Two novel GPCR-type G proteins are abscisic acid receptors in Arabidopsis. Cell 136, 136-148 (2009).

12. $\mathrm{Ma}$, $\mathrm{Y}$. et al. Regulators of $\mathrm{PP} 2 \mathrm{C}$ phosphatase activity function as abscisic acid sensors. Science 324, 1064-1068 (2009).

13. Park, S. Y. et al. Abscisic acid inhibits type $2 C$ protein phosphatases via the PYR PYL family of START proteins. Science 324, 1068-1071 (2009).

14. Fujii, $H$. et al. In vitro reconstitution of an abscisic acid signalling pathway. Nature 462, 660-664 (2009).

15. Eulgem, T., Rushton, P. J., Robatzek, S. \& Somssich, I. E. The WRKY superfamily of plant transcription factors. Trends Plant Sci. 5, 199-206 (2000).

16. Rushton, P. J., Somssich, I. E., Ringler, P. \& Shen, Q. J. WRKY transcription factors Trends Plant Sci. 15, 247-258 (2010).

17. Finkelstein, R. R., Wang, M. L., Lynch, T. J., Rao, S. \& Goodman, H. M. The Arabidopsis abscisic acid response locus AB/4 encodes an APETALA2 domain protein. Plant Cell 10, 1043-1054 (1998).

18. Lopez-Molina, L. \& Chua, N. H. A null mutation in a bZIP factor confers ABAinsensitivity in Arabidopsis thaliana. Plant Cell Physiol. 41, 541-547 (2000).

19. Shang, Y. et al. The Mg-chelatase $\mathrm{H}$ subunit of Arabidopsis antagonizes a group of WRKY transcription repressors to relieve ABA-responsive genes of inhibition. Plant Cell 22, 1909-1935 (2010).

20. Ren, X. Z. et al. ABO3, a WRKY transcription factor, mediates plant responses to abscisic acid and drought tolerance in Arabidopsis. Plant J. 63, 417-429 (2010).

21. Jiang, W. \& Yu, D. Arabidopsis WRKY2 transcription factor mediates seed germination and postgermination arrest of development by abscisic acid. BMC Plant Biol. 9, 1471-2229 (2009).

22. Ding, Z. J. et al. WRKY 41 controls Arabidopsis seed dormancy via direct regulation of $A B B 3$ transcript levels not downstream of $A B A$. Plant J. 79, 810-823 (2014)

23. Chen, L., Zhang, L., Li, D., Wang, F. \& Yu, D. WRKY 8 transcription factor functions in the TMV-cg defense response by mediating both abscisic acid and ethylene signaling in Arabidopsis. Proc. Natl Acad. Sci. USA 110, E1963-E1971 (2013).

24. Huang, Y., Feng, C. Z., Ye, Q., Wu, W. H. \& Chen, Y. F. Arabidopsis WRKY6 transcription factor acts as a positive regulator of abscisic acid signaling during seed germination and early seedling development. PLoS Genet. 12, e1005833 (2016).
25. Hu, Y. X., Wang, Y. H., Liu, X. F. \& Li, J. Y. Arabidopsis RAV1 is down-regulated by brassinosteroid and may act as a negative regulator during plant development. Cell Res. 14, 8-15 (2004).

26. Woo, H. R. et al. The RAV1 transcription factor positively regulates leaf senescence in Arabidopsis. J. Exp. Bot. 61, 3947-3957 (2010).

27. Feng, C. Z. et al. Arabidopsis RAV1 transcription factor, phosphorylated by SnRK2 kinases, regulates the expression of $A B|3, A B| 4$, and $A B 15$ during seed germination and early seedling development. Plant J. 80, 654-668 (2014).

28. Chen, $H$. et al. Roles of Arabidopsis WRKY18, WRKY40 and WRKY60 transcription factors in plant responses to abscisic acid and abiotic stress. BMC Plant Boil. 10, 281 (2010)

29. Lee, S. C. \& Luan, S. ABA signal transduction at the crossroad of biotic and abiotic stress responses. Plant Cell Environ. 35, 53-60 (2012).

30. Wei, W. et al. Ectopic expression of FWWRKY42, a WRKY transcription factor from the diploid woodland strawberry (Fragaria vesca), enhances resistance to powdery mildew, improves osmotic stress resistance, and increases abscisic acid sensitivity in Arabidopsis. Plant Sci. 275, 60-74 (2018).

31. Morozova, O., Hirst, M. \& Marra, M. A. Applications of new sequencing technologies for transcriptome analysis. Annu. Rev. Genom. Hum. Genet. 10 135-151 (2009).

32. Mutz, K. O., Heilkenbrinker, A., Lönne, M., Walter, J. G. \& Stahl, F. Transcriptome analysis using next-generation sequencing. Curr. Opin. Biotechnol. 24, 22-30 (2013).

33. Sudhagar, A., Kumar, G. \& El-Matbouli, M. Transcriptome analysis based on RNA-seq in understanding pathogenic mechanisms of diseases and the immune system of fish: a comprehensive review. Int. J. Mol. Sci. 19, 245 (2018).

34. Dong, $\mathrm{H}$. et al. Transcriptome analysis of soybean WRKY TFs in response to Peronospora manshurica infection. Genomics. (2018). https://doi.org/10.1016/j. ygeno.2018.09.014

35. Cheng, H. \& Wang, S. The important player of rice-pathogen interactions: WRKY-type transcription factors. Sci. Sin. Vitae 44, 784-793 (2014).

36. Liu, Q. et al. OsWRKY67 positively regulates blast and bacteria blight resistance by direct activation of PR genes in rice. BMC Plant Biol. 18, 257 (2018).

37. Zhang, L. L. et al. Overexpression of VaWRKY14 increases drought tolerance in Arabidopsis by modulating the expression of stress-related genes. Plant Cell Rep. 37, 1159-1172 (2018).

38. Zhang, Y. et al. CsWRKY46, a WRKY transcription factor from cucumber, confers cold resistance in transgenic-plant by regulating a set of cold-stress responsive genes in an ABA-dependent manner. Plant Physiol. Biochem. 108, 478-487 (2016).

39. Ullah, A., Sun, H., Yang, X. \& Zhang, X. A novel cotton WRKY gene, GhWRKY6like, improves salt tolerance by activating the ABA signaling pathway and scavenging of reactive oxygen species. Physiol. Plant. 162, 439-454 (2018).

40. $\mathrm{Ma}, \mathrm{Q}$. J. et al. An apple CIPK protein kinase targets a novel residue of AREB transcription factor for ABA-dependent phosphorylation. Plant Cell Environ. 40, 2207-2219 (2017)

41. Kang, J. Y., Choi, H. I., Im, M. Y. \& Kim, S. Y. Arabidopsis basic leucine zipper proteins that mediate stress-responsive abscisic acid signaling. Plant Cell 14, 343-357 (2002).

42. Kim, S., Kang, J. Y., Cho, D. I., Park, J. H. \& Kim, S. Y. ABF2, an ABRE-binding bZIP factor, is an essential component of glucose signaling and its overexpression affects multiple stress tolerance. Plant J. 40, 75-87 (2004).

43. Apel, K. \& Hirt, H. Reactive oxygen species: metabolism, oxidative stress, and signal transduction. Annu. Rev. Plant Biol. 55, 373-399 (2004).

44. Pei, Z. M. et al. Calcium channels activated by hydrogen peroxide mediate abscisic acid signalling in guard cells. Nature 406, 731 (2000).

45. Delledonne, M., Zeier, J., Marocco, A. \& Lamb, C. Signal interactions between nitric oxide and reactive oxygen intermediates in the plant hypersensitive disease resistance response. Proc. Natl Acad. Sci. USA 98, 13454-13459 (2001).

46. Asada, K. Production and scavenging of reactive oxygen species in chloroplasts and their functions. Plant Physiol. 141, 391-396 (2006).

47. Parcy, F. et al. Regulation of gene expression programs during Arabidopsis seed development: roles of the $A B / 3$ locus and of endogenous abscisic acid Plant Cell 6, 1567-1582 (1994).

48. Lopez-Molina, L., Mongrand, S., McLachlin, D. T., Chait, B. T. \& Chua, N. H. ABI5 acts downstream of $\mathrm{AB} 33$ to execute an $\mathrm{ABA}$-dependent growth arrest during germination. Plant J. 32, 317-328 (2002).

49. Shu, $\mathrm{K}$. et al. ABI4 regulates primary seed dormancy by regulating the biogenesis of abscisic acid and gibberellins in. Arab. PLoS Genet. 9, e1003577 (2013). 
50. Shu, $K$. et al. $A B \mid 4$ regulates the floral transition independently of $A B I 5$ and ABI3. Mol. Biol. Rep. 45, 2727-2731 (2018).

51. Zhang, X., Garreton, V. \& Chua, N. H. The AIP2 E3 ligase acts as a novel negative regulator of $\mathrm{ABA}$ signaling by promoting $\mathrm{AB} / 3$ degradation. Genes Dev. 19 1532-1543 (2005).

52. Bossi, F. et al. The Arabidopsis ABA-INSENSITIVE (ABI) 4 factor acts as a central transcription activator of the expression of its own gene, and for the induction of ABI5 and SBE2. 2 genes during sugar signaling. Plant J. 59, 359-374 (2009).

53. Lopez-Molina, L., Mongrand, S., Kinoshita, N. \& Chua, N. H. AFP is a nove negative regulator of $\mathrm{ABA}$ signaling that promotes $\mathrm{AB} / 5$ protein degradation. Genes Dev. 17, 410-418 (2003).

54. Gao, S. et al. ABF2, ABF3, and ABF4 promote ABA-mediated chlorophyll degradation and leaf senescence by transcriptional activation of chlorophyll catabolic genes and senescence-associated genes in Arabidopsis. Mol. Plant 9 , 1272-1285 (2016).

55. Nishimura, N. et al. Structural mechanism of abscisic acid binding and signaling by dimeric PYR1. Science 326, 1373-1379 (2009).
56. Santiago, J. et al. The abscisic acid receptor PYR1 in complex with abscisic acid Nature 462, 665-668 (2009).

57. Su, T. et al. WRKY42 modulates phosphate homeostasis through regulating phosphate translocation and acquisition in Arabidopsis. Plant Physiol. 4, 1579-1591 (2015).

58. An, J. P. et al. Ectopic expression of an apple cytochrome P450 gene MdCYPM1 negatively regulates plant photomorphogenesis and stress response in Arabidopsis. Biochem. Bioph. Res. Co. 483, 1-9 (2017).

59. Zhao, Q. et al. Overexpression of MdbHLH104 gene enhances the tolerance to iron deficiency in apple. Plant Biotechnol. J. 14, 1633-1645 (2016).

60. Hu, D. G. et al. MdMYB1 regulates anthocyanin and malate accumulation by directly facilitating their transport into vacuoles in apples. Plant Physiol. 170, 1315-1330 (2016)

61. Xie, X. B. et al. The bHLH transcription factor MdbHLH3 promotes anthocyanin accumulation and fruit colouration in response to low temperature in apples. Plant Cell Environ. 35, 1884-1897 (2012). 